\title{
Coronary stent rupture with infected false aneurysm and atrial fistula formation.
}

\author{
Mouhammad Kanj ${ }^{1}$, Mohammad Jomaa ${ }^{2}$, Roula Moucadie ${ }^{2}$, Moussa Abi Ghanem², and \\ Ziad Mansour ${ }^{2}$ \\ ${ }^{1}$ Lebanese University Faculty of Medical Sciences \\ ${ }^{2}$ Lebanese Hospital Geitaoui
}

May 5, 2021

\begin{abstract}
Infectious complications after percutaneous transluminal coronary angioplasty are uncommon and can occur at any point of time leading to high morbidity and mortality. We report a case of delayed coronary artery stent infection and rupture, with epicardial infected false aneurysm formation, and right coronary artery to right atrium fistula formation, presenting after one month of pyrexia.
\end{abstract}

\section{Introduction:}

Percutaneous Coronary Intervention (PCI) with stent placement is a well-established treatment for coronary artery stenosis. Although infectious complications are rare, it may appear at any time and be a life-threatening condition. Early recognition and institution of appropriate medical and surgical therapy is vital for patient survival. We report a case of a right coronary artery stent infection seven years after implantation. The patient also had coronary rupture with an infected false aneurysm, and a fistula to the right atrium.

\section{Case report:}

A 72-year-old male patient was referred to our hospital for one-month history of high-grade fever. The patient had multiple comorbidities including: end-stage kidney disease on haemodialysis in the last six months through a dialysis catheter, diabetes mellitus, and chronic obstructive pulmonary disease. He also had coronary artery disease (CAD) with a stent implantation in the right coronary artery (RCA) seven years before presentation, that was complicated with a hemopericardium and evacuated by a pericardial window. In addition, he had a complete atrio-ventricular block with a dual chamber pacemaker implantation. Physical examination revealed a holosystolic murmur at the left lower sternal border and severe bilateral lower limb oedema. Laboratory workup revealed leukocytosis (wbc $12700 \mathrm{c} / \mathrm{ul}$ ). Blood cultures were obtained. Transthoracic echocardiography showed decreased left ventricular ejection fraction (35\%), severely decreased right ventricular function, grade $4 / 4$ tricuspid regurgitation, $15-\mathrm{mm}$ mobile vegetation in the right atrium (RA), and a large saccular aneurysm on the RCA. CT angiography revealed a $58 \times 47 \mathrm{~mm}$ collection crossed by the RCA that has a thrombosed wall of $9 \mathrm{~mm}$ thickness (figure 1). Coronary angiography showed triple vessel CAD with extravasation of the contrast material through the RCA (video 1). He was prepared for surgery and had to be started on low dose norepinephrine due to hypotension. Under cardio-pulmonary bypass and after aortic cross-clamping, the false aneurysm was opened, pus came out; samples were sent to culture. Fibrin membranes and old clots were entirely removed (video 2). The RCA was completely avulsed; it was suture ligated (figure 2.A). The whole pouch was washed using povidone iodine, normal saline, and oxygen peroxide. The lateral wall of the RA seemed ruptured with creation of a fistula. A right atriotomy 
was performed. The fistula was found and debrided (figure 2.B). It was closed using a pericardial patch. De Vega technique was used for tricuspid valve annuloplasty, then CABG was done using saphenous vein grafts to the left anterior descending and the obtuse marginal arteries. Weaning of the bypass needed high doses of vasopressors. The patient was transferred to the cardiac surgery unit in a frail hemodynamic condition; three hours later, he developed severe sepsis resistant to maximal medical therapy, and unfortunately passed away.

The preoperative blood cultures grew MRSA. The intraoperative purulent secretions grew the same pathogen.

\section{Discussion}

Infective complications are rare after PCI. Less than thirty cases of coronary stent infection have been reported in the literature, most occurring during the four weeks following the procedure. In only few cases it did occur late. Staphylococcus aureus is by far the most common pathogen causing septic complications after PTCA [1]. Almost all untreated infected aneurysms in case of stent infection eventually lead to rupture $[2]$.

Our patient presented seven years after PCI with an epicardial abscess. The exact mechanism behind this complication remains unclear. The most likely hypothesis is having bacteraemia that took origin from the dialysis catheter that seeded in the right coronary stent.

Coronary fistula in stent infection seldom happens. Only two similar cases were found in the literature $[3,4]$. Our case was unique in its late presentation, presence of coronary rupture, infected false aneurysm, and right atrial fistula formation.

Whatever the mechanism of stent infection is, it is always a life-threatening condition; all aseptic precautions should be taken to prevent this iatrogenic infection. We believe that the delay in surgical therapy in addition to the multiple comorbidities and the poor hemodynamic status our patient had, resulted in his poor outcome.

\section{Conclusion}

Stent infection should always be considered whenever a patient presents with systemic infection after PCI. A faster surgical approach should be warranted in these unstable patients, exclusive medical therapy should be discussed on a case by case basis.

\section{Author contributions:}

Mouhammad Kanj: Concept/design, Data analysis/interpretation, Drafting article, Critical revision of article, Data collection.

Mohammad Jomaa: Concept/design, Data analysis/interpretation.

Roula Moucadie: Concept/design, Data analysis/interpretation, Data collection.

Moussa Abi Ghanem: Data analysis/interpretation, Data collection.

Ziad Mansour: Concept/design, Data analysis/interpretation, Drafting article, Critical revision of article, Approval of article, Data collection.

\section{References:}

[1] Lalwala D, Deshmukh M, Mehta S, Nagvekar V. Coronary Stent Infection: A Rare but Severe Complication of Percutaneous Coronary Intervention. J Cardiovasc Dis Diagnosis 2018;06.

[2] Elieson Marc, MixonTimothy, Carpenter John. Coronary Stent Infections A Case Report and Literature Review. Tex Hear Inst J 2012;39:884-9.

[3] Shafer K, Toma C, Galdys A. A common pathogen in an uncommon site: coronary artery stent meticillinresistant Staphylococcus aureus infection. JMM Case Reports 2017;4. 
[4] Sangolkar R, Ketana VRR, Sastry BKS. Coronary artery stent infection presenting as coronary cameral fistula: a case report. Eur Hear J - Case Reports 2018;2.

\section{Legends:}

Figure 1: CT angiography showing the pseudoaneurysm (dashed arrow), the RCA (black arrow) and the mural thrombus (white arrow).

Figure 2: (A) RCA free edge (white arrow) suture ligated; cavity of the pseudoaneurysm (black arrow). (B) Right angle clamp passing through the RA fistula reaching the pseudoaneurysm (black arrow); RA pacemaker lead (white arrow).

Video 1: Extravasation of contrast through the RCA.

Video 2: Debridement of the pseudoaneurysm.

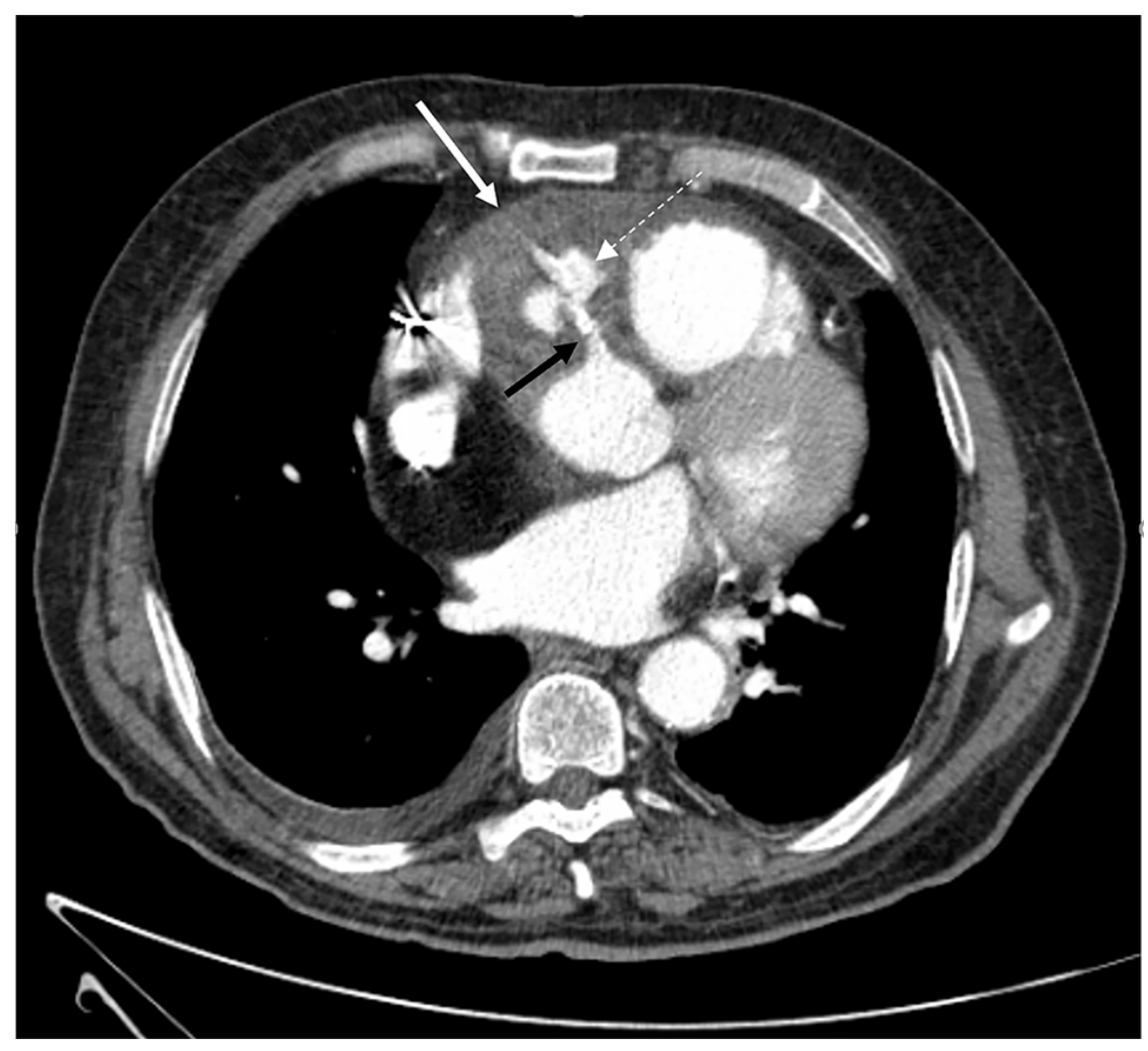



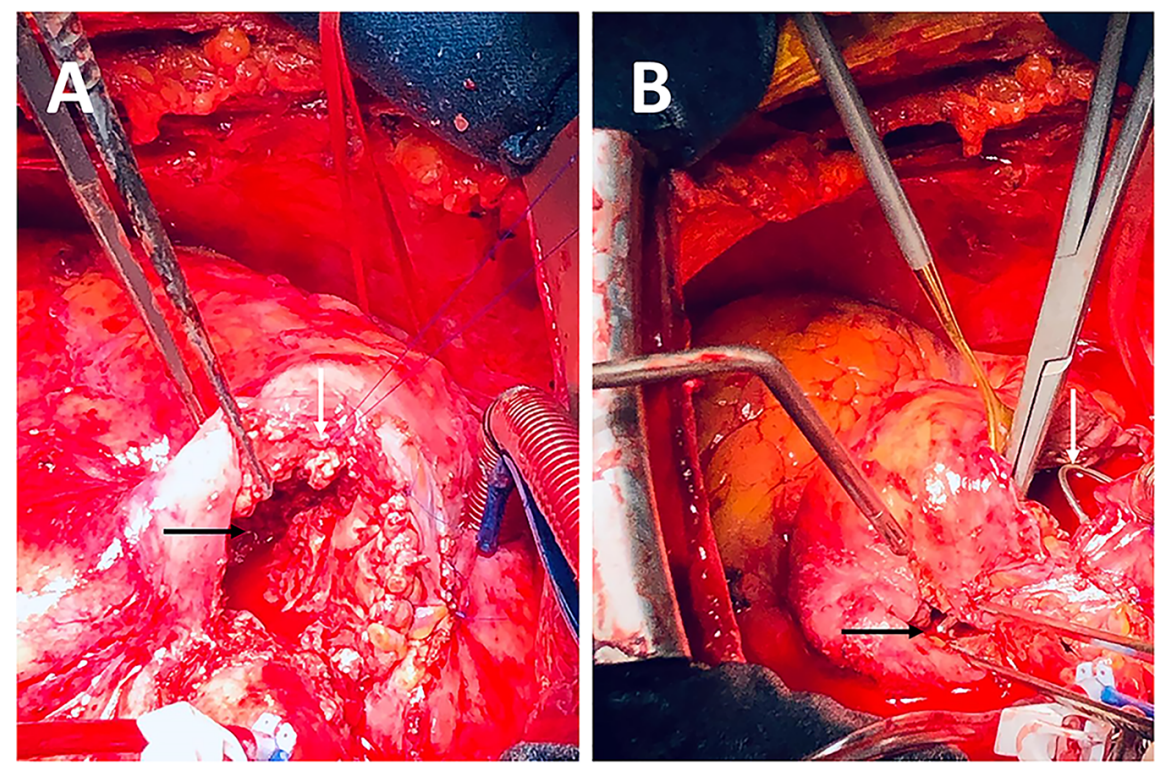